\title{
Afterheat Storage and Generating Device Based on the Stirling Generator
}

\author{
Shuguo Zhang ${ }^{a}$, Mengshu Shi ${ }^{b}$ \\ School of North China Electric Power University, BaoDing 071000, China \\ a13930865901@163.com, bshimengshu@ncepuenactus.cn
}

Keywords: Stirling Generator; Laptop; Afterheat Generation; Heat Conduction Liquid; Copper Pipe.

\begin{abstract}
Nowadays, as a useful tool, the laptop is commonly used in our daily life. However, due to the poor heat dispersion capability, the working efficiency of the laptop has been decreased a lot. In order to solve this problem, many users tend to purchase an extra radiator for the laptop, which is a waste of money and resources. Based on the status, we came up with the idea to recycle the afterheat. Considering the actual need of users and the technical support we possess, we plan to improve and extend the function of the current Stirling generator and lower its working temperature to match the laptop. Based on the principle of Stirling generator and combined with the large-capacity battery, the electricity generated from the afterheat will be stored in the battery as the portable power bank of the laptop. Through this process, we successfully produce renewable energy by reusing afterheat from the laptop.
\end{abstract}

\section{Introduction}

With the further research of environment-friendly electronic devices, researchers have devoted their efforts in the development of them, but an industry chain has not been formed. Starting with the laptop, our team designs the device that is capable of absorbing the afterheat to generate electricity. On the one hand, we solve the problem of poor heat dispersion capability of the laptop. On the other hand, we actually send the heat back to the laptop in the form of usable energy. Although there are countless electronic devices in the market, our device is the first one of its kind. Laptop afterheat generating is a brand new direction of producing renewable energy, which can guarantee the promising future of our project.

\section{Technical Specialty}

Our device is designed on the basis of the traditional Stirling generator. We improve the devices to satisfy the need of the laptop and combine it with the portable power bank. When the laptop is working, the device will absorb the heat from the laptop, generate electricity and store it in the battery. When the laptop needs charging, the device will serve as a portable power source.

The device consists of 5 parts: (1) Strong silicone sleeve; (2) Gas conductive foam; (3) Heat conductive copper pipe (with heat conduction liquid); (4) Small-scale Stirling generator; (5) Storage battery.

The requirements of each part are as follows:

A .Strong silicone sleeve: The silicone sleeve is a device that connects the gas conductive foam with the laptop. The silicone sleeve is made of heat insulating toughness plastics, which can conduct the heat into the copper pipe. In the meantime, the sizes of this powerful acetabulum are various to suit different shapes of exhaust holes and the sleeve itself is convenient to be carried with your laptop.

B.Gas conductive foam: The thermal conductivity of the foam is low and its thermal performance is satisfying, which make it excellent in decreasing the heat loss.

C.Heat conductive copper pipe: The thermal conductivity of copper is high and the copper pipe is placed inside the foam. It is used to maintain the heat conductive oil.

D.Heat conductive liquid: The heat conductive liquid is a kind of oil that objectively conducts the heat. Its temperature is balanced and it will be continuously heating the generator. 
E.Stirling generator: The generator is connected to the storage battery. It is motivated by the heat from the laptop and will generate electricity.
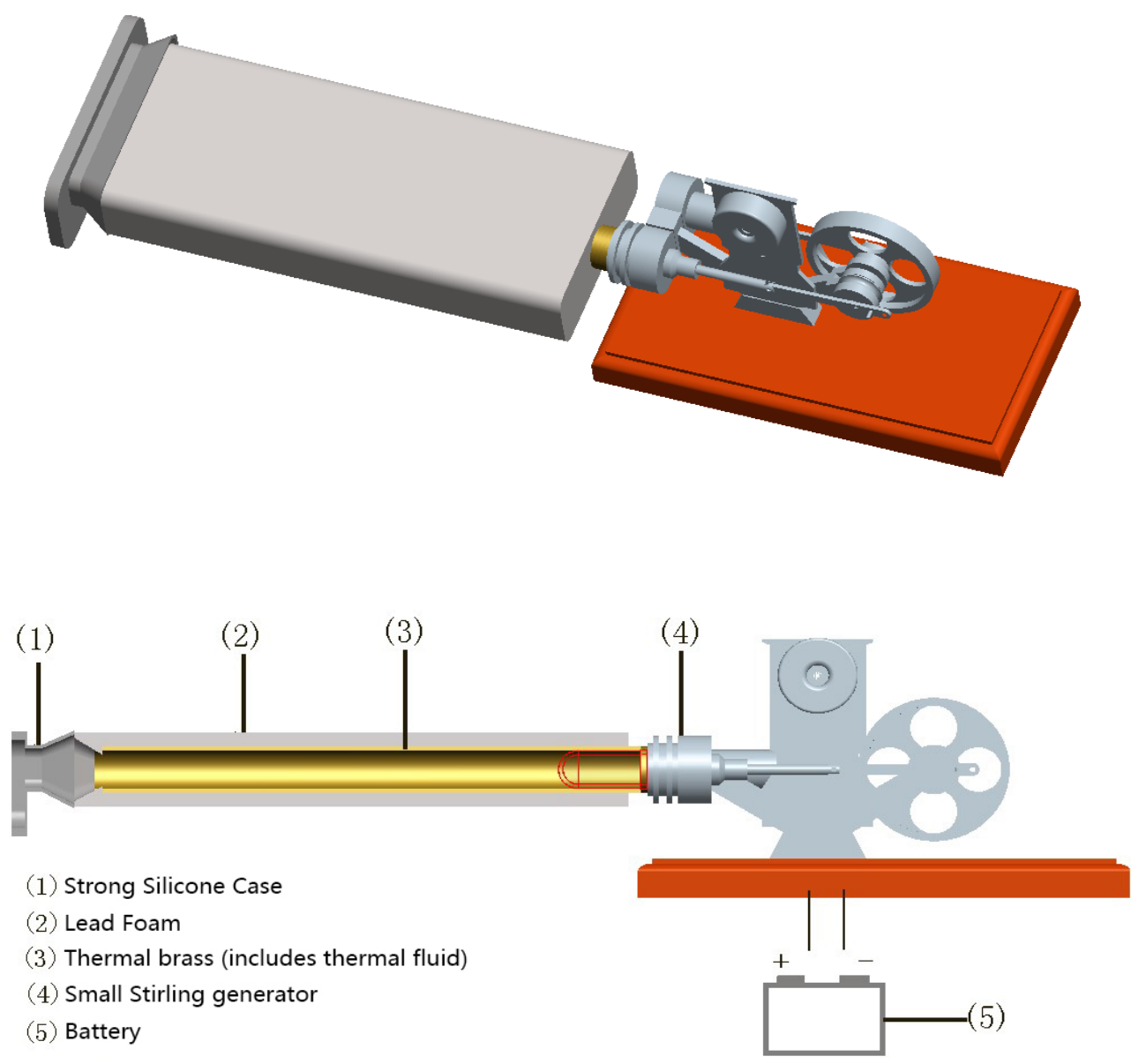

Operational Principle: The silicone sleeve is well connected to the exhaust hole of the laptop to make sure that most of the heat is conducted into the foam. The heat conductive copper pipe is filled with heat conductive liquid and is surrounded by the foam. The liquid in the pipe serves to balance the temperature and continuously heat the glass tube of the Stirling generator. The generator will firstly transform heat into mechanical energy then into electricity and store the electricity in the storage battery,

\section{Experiments:}

The measurement of the heat loss of the exhaust hole.The forced convection of the exhaust hole is formed by the fan inside the laptop. Therefore, the measurement of the heat loss consists of 3 parts:

A.The measurement of the wind speed

B.The measurement of the area of the exhaust hole

C.The measurement of the airflow dynamic temperature

The measurement of the wind speed:

We use a hot wire anemometer to measure the wind speed.

We can read the current wind speed from the hot wire anemometer. We will get the average wind speed by averaging all the 5 data we gather. 


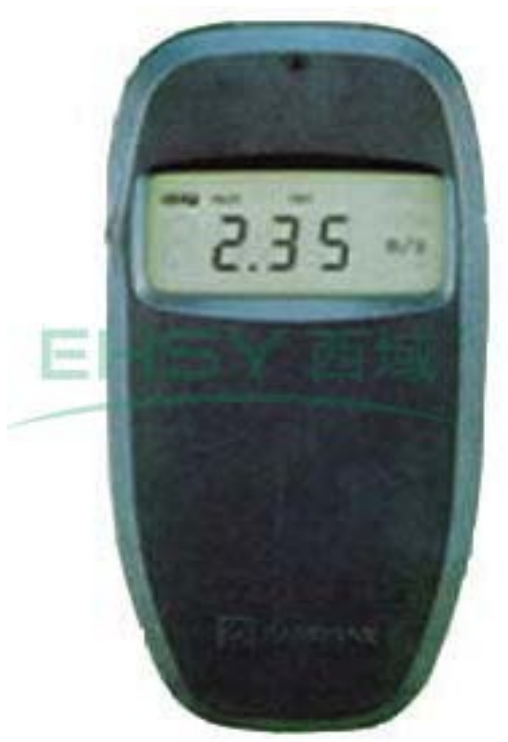

The measurement of the airflow dynamic temperature

Technically, our device is definitely a model of the energy-saving and low-emission. Influenced by the concept of environmental friendliness and green life, we take an interest in afterheat generation. The material of our device is easy to get and it's very simple to set up the device.

$$
\bar{h}=\frac{\overline{N u_{L}} k}{L}
$$

Economically, our device can continuously generate electricity as the laptop works and it's capable of motivating low-energy-consuming devices. A 15 watt LED light consumes 4.5 kilowatt hour of electricity in 30 days when working 10 hours a day. However, using a laptop with our device, under the same circumstance, only 0.45 kilowatt hour of electricity is consumed. Our device performs dramatically well in energy saving, which guarantees the prospect of it. Conservatively estimating, there are 350 million netizens in China. After using our device, 1 billion kilowatt hour of electricity will be saved per month. Calculating on the basis of 0.5 yuan per kilowatt hour of electricity, we will save 500 million yuan per month in China. If all netizens use our device all over the world, 3 billion kilowatt hour of electricity and 1.5 billion yuan will be saved monthly.

\section{Prospect}

Our project is devoted to solving the waste of energy and achieving the cyclic utilization of energy. Our device solves the problem of poor heat dispersion capability of the laptop and extends the operation life span of them. Because we are the first to invent the device, a large potential market is given to us. Our device stands for the concept of energy saving and environmental protection and will lead to the development of environmental-friendly industry. Nowadays, with the rapid development of electronic communication, the development of the computer as a vitally useful tool will be unlimited. In the meantime, the waste of resources and energy is increasing day by day and it becomes a major concern of our society. Besides, with the development of economy, people tend to be in pursuit of a better life standard, which becomes an advantage for our device.

\section{Conclusion}

Our device is fully qualified for the concept of energy saving and low emission. Apart from saving the energy, it transforms the energy at the same time. The key part of the device is the heat conduction and we use good conductor of heat, which can absorb the heat with high efficiency. Our technology is competitive both commercially and environmentally and reflecting the theme of green development. Our device cuts down the heat emission and generates electricity, which is really an innovative product. 
In the meantime, the potential customers of our device are countless. All laptop users can be users of our device. Through market research, we discover that most of the laptop users we've surveyed express keen interest in our device. What's more, the price of the device is inexpensive and affordable for most people. We are confident about its future.

Our device is a brand new discovery in radiation devices and it serves as a precious aspect in minimalizing industrial afterheat generating system.

\section{References}

[1] Lifu Jun, Ma Yuan Qing, Li Bao, the Wang Yong. "Large refrigeration equipment cogeneration technology to explore" [J]. Energy Saving,2013,12-12.

[2] Lei Feng "HTF techniques and its application" [J] oil, 2005,20 (6): 58-62.

[3] Shao Yun, "study pure low temperature waste heat power generation control technology," 2010-05-20

[4] Wang wealth "Study on low temperature waste heat power generation model" 2003-03-01

[5] Yin Gang Wu Fangsong Zhang Lizhi 'characteristics and trends of low temperature waste heat power generation technology to explore "2011-03-25

[6] Li Shengmei "cogeneration automatic control system," 2012-05-01

[7] Wang chrysanthemum "application of co generation systems Intelligent Controller" 2012-05-01

[8] Xu Qingbo "pure low temperature waste heat power generation and application of technical and economic evaluation" 2010-05-04

[9] Cheng Rongrong "Notebook heat pipe cooling system structural parameters and characteristics analysis" 2014-12-20

[10]Zhang Shuqing[Study of the free-piston Stirling generator control system"] 2014-06-01 